\title{
Modelo experimental de vasectomia laparoscópica em $\operatorname{ratos}^{1}$
}

\author{
Lúcio Couto de Oliveira $\mathrm{Jr}^{2}$ \\ Fabrício Mascarenhas de Oliveira ${ }^{3}$ \\ Vanessa Alves Costa ${ }^{3}$ \\ Mario Castro Carreiro ${ }^{4}$ \\ Pedro Guimarães 5
}

\begin{abstract}
Oliveira Jr LC, Oliveira FM, Costa VA, Carreiro MC, Guimarães P. Modelo experimental de vasectomia laparoscópica em ratos. Acta Cir Bras [serial online] 2003 Set-Out;18(5). Disponível em URL:http://www.scielo.br/acb.
\end{abstract}

RESUMO - Objetivo: Descrever um modelo experimental de vasectomia laparoscópica em ratos. Métodos: Foram utilizados 35 ratos Rattus norvegicus albinus da linhagem Wistar. Após a anestesia, os ratos foram submetidos ao pneumoperitôneo através da agulha de Veress com PCO2 de $7 \mathrm{mmHg}$. Depois, foram transpassados pela parede abdominal um trocater de $11 \mathrm{~mm}$ de espessura e dois trocateres com $5 \mathrm{~mm}$ de espessura. Através destes foram introduzidos na cavidade abdominal à ótica (10mm) e as pinças utilizadas na dissecção, cauterização e secção do canal deferente e seus vasos. Resultados: Durante o ato operatório dois animais $(5,74 \%)$ apresentaram sangramento, contido com cauterização. Ocorreu um óbito na indução anestésica $(2,85 \%)$ e outro $(2,85 \%)$ no quarto dia pós-operatório de causa não identificada. Após 08 dias havia 33 ratos vivos $(94,28 \%)$. Conclusão: Neste modelo, o material cirúrgico é o mesmo utilizado em humanos e são aplicados todos os procedimentos básicos da cirurgia laparoscópica, tornando-o factível para o treinamento de acadêmicos, médicos residentes e cirurgiões.

DESCRITORES - Cirurgia laparoscópica. Vasectomia. Ratos.

\section{Introdução}

A cirurgia laparoscópica tem apresentado grande aplicabilidade em modelos experimentais. Técnicas de vasectomia escrotais e transabdominais em animais são comparadas em estudo ${ }^{1}$ que avalia as alterações testiculares decorrentes dessas cirurgias. No ano de 1987 foi realizado um estudo quantitativo dos efeitos da vasectomia na espermatogênese em ratos. Um modelo experimental de vasectomia laparoscópica intraabdominal em lhamas e alpacas usando eletrocautério e seccionando 2 a $3 \mathrm{~cm}$ do ducto deferente ${ }^{2}$ foi descrito em 1991; os autores consideraram a técnica simples, segura e rápida ${ }^{3}$, sendo esta a mesma conclusão de outros pesquisadores que esterilizaram 5 cães através de uma vasectomia laparoscópica ${ }^{4}$. Em 1998 foi descrita uma técnica de vasectomia laparoscópica para ratos utilizando instrumentais laparoscópicos de pequeno porte e um pneumoperitôneo de $7 \mathrm{mmHg}$ com insuflação de $\mathrm{CO} 25$.

Os modelos experimentais de cirurgia laparoscópica evoluíram de animais de médio porte como cães e porcos ${ }^{4,6}$ até animais de laboratório como coelhos e

1. Trabalho realizado na Escola Bahiana de Medicina e Saúde Pública (EBMSP).

2. Médico da EBMSP.

3. Acadêmicos da EBMSP.

4. Professor Adjunto IV da Universidade Federal da Bahia (UFBA). Coordenador da Disciplina de Técnica Operatória e Cirurgia Experimental da Universidade Federal da Bahia (UFBA)

5. Professor Adjunto da Disciplina de Técnica Operatória e Cirurgia Experimental da Universidade Federal da Bahia (UFBA). Doutor pela Universidade Federal de São Paulo - Escola Paulista de Medicina da (UNIFESP - EPM). 
ratos ${ }^{5,7-11}$. Estes permitiram redução de custos comparando com os animais de maior porte e uso mais extensivo em trabalhos científicos bem como no treinamento de profissionais.

\section{Métodos}

Os experimentos foram aprovados pela comissão de ética da Escola Bahiana de Medicina e Saúde Pública (EBMSP).

Para o presente estudo foram utilizados 35 ratos Rattus norvegicus albinus da linhagem Wistar, procedentes do biotério da E.B.M.S.P. - BA, pesando $250+/-50 \mathrm{~g}$, com idade média de três meses sendo todos machos. Os animais foram colocados em gaiolas individuais, medindo $20 \times 30 \times 40 \mathrm{~cm}$, aclimatados durante 15 dias a uma temperatura de $25^{\circ} \mathrm{C}$, em períodos de 12 horas luz e 12 horas escuridão com ração e água livres.

\section{Pré-operatório}

Cerca de oito horas antes do procedimento, os animais foram colocados em jejum e anestesiados por via intra-peritoneal com cetamina $(7 \%)$ e xilasina $(0.3 \%)$ na proporção de $2: 1 \mathrm{ml}$, com $0,2 \mathrm{ml} / 100 \mathrm{~g}$ de peso da solução. Em seguida, foram posicionados na mesa cirúrgica em decúbito dorsal, tendo suas extremidades fixadas com hastes de borracha, tricotomizados e submetidos à antissepsia.

\section{Ato operatório}

Após a colocação dos campos, foi feita uma incisão com $0.5 \mathrm{~cm}$ de comprimento com lâmina de bisturi frio $\mathrm{n}^{\circ} 15$, a uma distância de $1 \mathrm{~cm}$ abaixo do rebordo costal esquerdo, em sua porção medial, abrangendo pele e aponeurose. Realizou-se a fixação da musculatura abdominal com as pinças de Allis, para tracionar a parede abdominal, com o intuito de facilitar a passagem da agulha de Veress a ângulo de $90^{\circ}$ através da mesma.

Foram feitos testes de segurança, conexão da mangueira do insuflador e início do pneumoperitôneo com PCO2 de 7 mmHg. ${ }^{5,7}$

A segunda incisão com $1,0 \mathrm{~cm}$ de comprimento foi realizada distando $1.0 \mathrm{~cm}$ abaixo do apêndice xifóide, na linha média abdominal, através da qual atingiu-se "às cegas" a cavidade abdominal com trocater de $11 \mathrm{~mm}$, que foi fixado com sutura em bolsa na pele do animal impedindo o escape de $\mathrm{CO} 2$ durante a cirurgia.

A óptica de $10 \mathrm{~mm}$ foi introduzida e, através da sua imagem ou "visão direta", a agulha de Veress foi retirada e um trocater com $5 \mathrm{~mm}$ introduzido em seu lugar. Então, uma terceira e última incisão foi feita distando $1.0 \mathrm{~cm}$ abaixo do rebordo costal direito em sua porção medial e medindo $0.5 \mathrm{~cm}$, através da qual, sob visão direta, um terceiro trocater com $5 \mathrm{~mm}$ de diâmetro foi posicionado.

Já na cavidade, o primeiro passo consistiu na redução do testículo direito do saco escrotal. As estruturas peri-testiculares foram então apreendidas delicadamente pelo cirurgião utilizando uma pinça atraumática e com uma pinça Maryland (Figura 1) foi feita a dissecção do ducto deferente e de seus vasos até que uma extensão de aproximadamente $2 \mathrm{~cm}$ do ducto e de seus vasos sanguíneos estivesse exposta e isolada de outras estruturas.

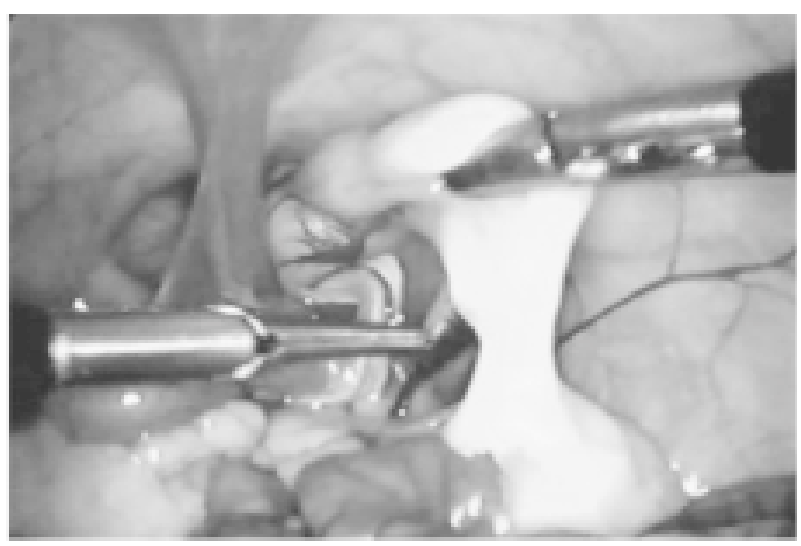

FIGURA 1 - Apreensão de estruturas peritestículares com pinça atraumática, permitindo dissecção do ducto deferente e de seus vasos com uma pinça Maryland.

A seqüência do procedimento consistiu na cauterização proximal e depois distal do deferente (Figura 2) sendo a primeira a $1 \mathrm{~cm}$ da junção do deferente com o epidídimo e a segunda a $0,5 \mathrm{~cm}$ da primeira; ${ }^{2}$ a cauterização é feita com a Maryland conectada ao eletrocautério.

O mesmo procedimento de cauterização é feito

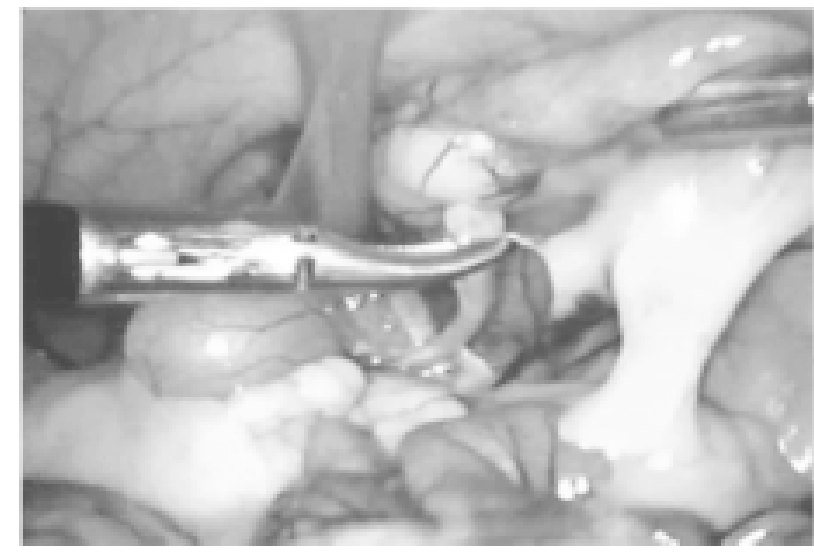

FIGURA 2 - Cauterização do ducto deferente. 
com os vasos sanguíneos. A Maryland foi substituída pela tesoura e foi feita a secção do deferente entre as duas cauterizações (Figura 3).

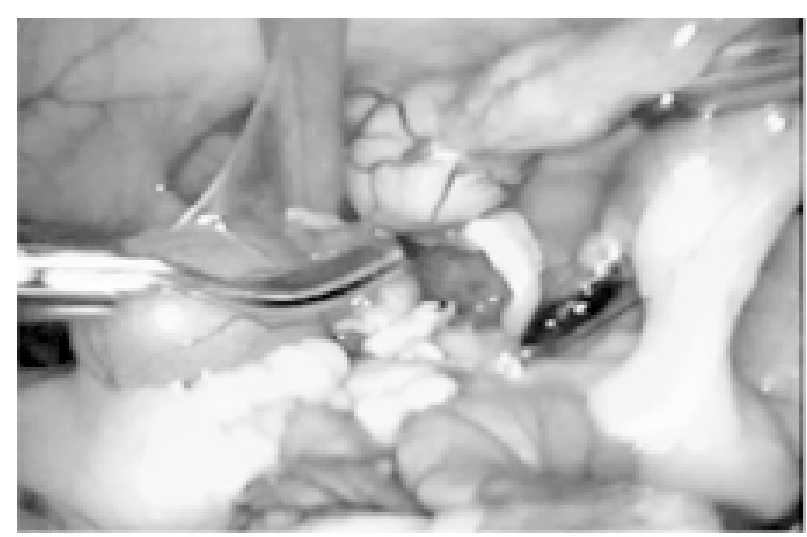

FIGURA 3 - Secção do deferente previamente dissecado e cauterizado.

\section{Pós-operatório}

Os animais foram re-colocados em gaiolas individuais e, observados durante oito dias, nas mesmas condições já citadas (ver método). Após um período de observação de oito dias os animais foram sacrificados em uma câmara fechada com éter etílico e submetidos a autópsia.

\section{Resultados}

O tempo operatório médio foi de $15+/-6$ minutos e em nenhum dos animais foi necessária a conversão para a cirurgia aberta.

Dos 35 animais, um morreu na indução anestésica $(2,85 \%)$ e dois $(5,74 \%)$ apresentaram sangramento no ato operatório no momento de dissecção dos vasos sanguíneos do deferente. O sangramento foi contido com cauterização da estrutura lesada e a cirurgia transcorreu sem outras intercorrências. Outro animal $(2,85 \%)$ foi a óbito no quarto dia pós-operatório de causa não identificada através da necropsia.

Após 08 dias havia 33 ratos vivos $(94,28 \%)$ e na necrópsia, não foram observadas deiscências de sutura, necrose, áreas de hemorragia e nem sinais de infecção em nenhum dos animais.

\section{Discussão}

A cirurgia laparoscópica tem mostrado um crescimento importante nas últimas décadas e hoje é indicada como procedimento de primeira escolha em muitos casos. A cirurgia experimental mostra-se como mecanismo fundamental para o treinamento e aperfeiçoamento de cirurgiões. ${ }^{8-13}$
$\mathrm{Na}$ literatura são encontrados relatos sobre a aplicação da laparoscopia em modelos experimentais, principalmente em porcos e cães. ${ }^{4,6}$ além de outros animais como lhamas e alpacas, ${ }^{3}$ no entanto, ainda são poucos os modelos em animais de laboratório como ratos ${ }^{10} \mathrm{e}$ coelhos. A vídeo cirurgia nestes animais de menor porte, apresenta as vantagens de um baixo custo, e da prática em larga escala de todos os aspectos de um procedimento minimamente invasivo com visão indireta bidimensional: apreensão, dissecção, cauterização e secção de estruturas orgânicas.

Neste estudo, utilizou-se o mesmo material da operação em humanos sem adaptação de nenhum instrumental. Com isso, as condições de treinamento tornam-se ainda mais eficazes. Como complicação do ato operatório, houve dois sangramentos na dissecção dos vasos do deferente que foram facilmente contidos através de cauterização e os animais não apresentaram outras intercorrências no pós-operatório.

\section{Conclusão}

O modelo apresentado neste trabalho mostra-se eficaz para que se procedam a vasectomias laparoscópicas em ratos com o objetivo de treinamento de acadêmicos, médicos residentes e até mesmo cirurgiões nesta crescente área que é aplicação de procedimentos minimamente invasivos no ato operatório.

Com uma técnica cirúrgica devidamente padronizada, o rato é um modelo simples, barato e eficaz.

\section{Referências}

1. Neaves WB. The rat testis after vasectomy. J. Reprod. Fertil 1974; 40:39-44

2. McDonald SW, Scothorne RJ. A quantitative study of the effects of vasectomy on spermatogenesis in rats. J Anat 1998;159:219-25.

3. Bravo PW, Sumar J. Evaluation of intra-abdominal vasectomy in llhamas e alpacas. J Am Vet Med Assoc 1991;199(9) 1164-6.

4. Silva LD, Onclin K, Donnay I, Verstegen JP. Laparoscopic vasectomy in the male dog. Reprod Fertil 1993; 47: 399-401.

5. Gutt CN, Riemer V, Brier C, Berguer,R, Paolucci V.Standardized technique of laparoscopic surgery in the rat. Dig Surg 1998;15:135-9.

6. Chiu AW, Chang LS, Birkett DH, Babayan RK. Changes in urinary output and electrolytes during gaseous and gasless laparoscopy. Urol Res 1996;24:361-6.

7. Berguer R, Cornelius T, Dalton $M$. The optimum pneumoperitoneum pressure for laparoscopic surgery in the rat model. Surg Endosc 1997;11: 915-8.

8. Filmar F, Gomel V, McComb PF. Operative laparoscopic versus open abdominal surgery: a comparative study on postoperative adhesion formation in the rat model. Fertil Steril 1987; 3:486-9.

9. Berguer R, Gutt CN. Laparoscopic colon surgery in a rat model: a preliminar report. Surg Endosc 1994;8:1195-7. 
10. Goldenberg A, Lobo EJ, Marcondes W et al. A proposition to videolaparoscopy in rats. Acta Cir Bras 1997;12(4):219-20.

11. Kalach AC, Baro MCH, Vorero NH, Marcovich DL, Rojas AC, Barrera RC, Chaba SS. La rata como modelo animal para la enseñanza e investigación de la cirugía laparoscópica. Cir Gen 1996;3: 221-7.
12. Berguer R, Gutt CN, Stiegmann GV. Laparoscopic surgery in the rat: description of a new technique. Surg Endosc $1993 ; 7: 345-7$

13. Giuffriga MC, Marquet RL, Kazemier G, Wittich P, Bouvy ND, Bruining HAS, Bonjer HJ: Laparoscopic splenectomy and nephrectomy in a rat model: description of a new technique. Surg Endosc 1997;11:491-4.

Oliveira Jr LC, Oliveira FM, Costa VA, Carreiro MC, Guimarães P. An experimental model of laparoscopic vasectomy in rats. Acta Cir Bras [serial online] 2003 Sept-Oct;18(5). Available from URL: http://www.scielo.br/acb.

ABSTRACT - Purpose: To describe a model of laparoscopic vasectomy in rats. Methods: 35 rats Rattus Norvegicus Albinus Wistar were used. The animals were anaesthetised and then the pneumoperitoneum was done with $\mathrm{PCO} 2$ of $7 \mathrm{mmHg}$ through a Veress needle. After that, a $11 \mathrm{~mm}$ trocar and two $5 \mathrm{~mm}$ trocars were inserted through the abdominal wall. The forceps used on dissections, cauterisation and section of the ductus deferens and its vessels and the $10 \mathrm{~mm}$ diameter laparoscope were introduced through the trocars. Results: In the course of surgery, two animals $(5,74 \%)$ presented bleeding which was easily stopped in both cases. One animal died at the anaesthetic induction $(2,85 \%)$ and another $(2,85 \%)$ died of an unidentified cause on the fourth observation day. After an eight-day observation period, 33 rats were alive $(94,28 \%)$. Conclusions: The surgical material is the same as that used in humans and all the basic procedures of laparoscopic surgery are applied, which makes it feasible in the training of students, residents and surgeons.

KEY WORDS - Laparoscopic surgery. Vasectomy. Rats.

Conflito de interesse: nenhum

Fonte de financiamento: nenhuma

Correspondência:

Lúcio Couto de Oliveira Júnior

Rua Prof. Augusto Machado, 330/404B

41910-070 Salvador - BA

luciocjr@uol.com.br

Data do recebimento: 27/06/2003

Data da revisão: 08/07/2003

Data da aprovação: 16/07/2003 